\title{
MODELO DE PROGRAMA DE CONTROL DE PARASITOSIS INTESTINALES Y NUTRICIÓN EN ESCOLARES DE ARGENTINA: INTEGRANDO DOCENCIA, EXTENSIÓN E INVESTIGACIÓN
}

MODELO DE PROGRAMA EM CONTROLE DE
PARASITISMO INTESTINAL E NUTRIÇÃO EM
ESTUDANTES DA ARGENTINA: INTEGRANDO
DOCENCIA, EXTENSÃO E INVESTIGAÇÃO

THE MODEL OF A PROGRAM FOR CONTROL OF INTESTINAL PARASITOSIS AND NUTRITION IN STUDENTS OF ARGENTINA: INTEGRATING TEACHING, OUTREACH AND RESEARCH

\author{
Betina Cecilia Pezzani* \\ Maria Laura Ciarmela** \\ Alicia Bibiana Orden ${ }^{* * *}$ \\ Marina Isla Larrain ${ }^{* * * *}$ \\ Diana Rosa***** \\ Alejandro J. Mariñelarena ${ }^{* * * * *}$ \\ Josefina Anabitarte (******* $^{* *}$ \\ Marta Cecilia Minvielle
}

\section{RESUMEN}

Presentamos un modelo de Programa que integra actividades de docencia, extensión e investigación, desarrollado en la Facultad de Ciencias Médicas de la Universidad Nacional de La Plata, Argentina denominado PROCOPIN (Programa de Control de las Parasitosis Intestinales y Nutrición). Este modelo de programa refleja la unión entre la institución universitaria, los profesionales de la salud pública regional y los directivos de establecimientos escolares para lograr el control de las infecciones parasitarias intestinales y mejorar las condiciones nutricionales de niños en condiciones de vulnerabilidad social. Con un significativo valor formativo para los estudiantes de nuestra facultad, integrando contextos y

\footnotetext{
*Bióloga. Universidad Nacional de La Plata (UNLP), La Plata - Argentina. E-mail: bpezzani@med.unlp.edu.ar

**Bióloga. Universidad Nacional de La Plata (UNLP), La Plata - Argentina. E-mail: ciarmela@med.unlp.edu.ar

***Antropóloga. Universidad Nacional de La Plata (UNLP), La Plata - Argentina. E-mail: aborden@conicet.gov.ar

****Bioquímica. Universidad Nacional de La Plata (UNLP), La Plata - Argentina. E-mail: marinaislalarrain@hotmail.com

*****Medica Veterinária. Universidad Nacional de La Plata (UNLP), La Plata - Argentina. E-mail: dianita_rosa@yahoo.com.ar

******Biólogo. Universidad Nacional de La Plata (UNLP), La Plata - Argentina. E-mail: alemar@ilpla.edu.ar

*******Aluna da Universidad Nacional de La Plata (UNLP), La Plata - Argentina. E-mail: josefinanabitarte@hotmail.es 
contenidos curriculares, articulando lógicas institucionales diversas, incentivando el trabajo en equipo y adquiriendo una mirada crítica sobre problemáticas socio-sanitarias.

Palabras clave: parasitosis intestinales; nutrición; escolares; Argentina

\title{
RESUMO
}

Apresentamos um modelo de Programa que integra atividades de docência, extensão e investigação, desenvolvido pela Faculdade de Ciências Médicas da Universidade Nacional de La Plata, Argentina denominado PROCOPIN (Programa de Controle de Parasitosis Intestinais e Nutrição). Este modelo de programa reflete a união entre a instituição universitária, os profissionais da saúde pública regional e diretores dos estabelecimentos escolares, para garantir o controle das infeções parasitárias intestinais e melhorar as condições nutricionais das crianças em condições de vulnerabilidade social. Com um valor de formação significativo para os estudantes da nossa faculdade, integrando contextos e conteúdos curriculares, articulando lógicas institucionais diversas, incentivando o trabalho de equipe e adquirindo uma visão crítica sobre problemas sócio-sanitários.

Palavras-chave: parasitose intestinal; nutrição; escola; Argentina

\begin{abstract}
This study aims to describe the model of a program that integrates teaching, outreach and research called Program for Control of Intestinal Parasitoses and Nutrition (PROCOPIN). It is developed at the Faculty of Medical Sciences of the National University of La Plata, Argentina. It reflects on the joint effort of public health professionals, school managers and the university to control intestinal parasitic infections and to improve the nutritional conditions of children in social vulnerability. It has a significant formative value for students of the faculty since it integrates contexts and curricular contents, articulates institutional logics, encourages team work and critical view on socio-sanitary problems.
\end{abstract}

Keywords: intestinal parasitoses; nutrition; school; Argentina.

\section{Introducción}

Muchos países en vías de desarrollo han experimentado un significativo descenso de la mortalidad infantil, en las últimas tres décadas. En Latinoamérica se sostiene un alto índice junto a problemas tales como la desnutrición, anemia e infecciones parasitarias que afectan especialmente el desarrollo físico e intelectual de los niños. Generalmente estas parasitosis son de curso crónico y van deteriorando lentamente al individuo de tal manera que no percibe la afectación de su organismo, hasta que alcanza niveles muchas veces irreversibles (Culha y Sangun, 2007; Hotez y col., 2008).

Estas infecciones intestinales son indicadores sensibles del estado de salud de las poblaciones. Está comprobado que factores ambientales, como la contaminación fecal de suelos y aguas y factores socioculturales como la falta de hábitos de higiene, el bajo nivel de instrucción, la carencia de instalaciones sanitarias adecuadas, el hacinamiento, el contacto con animales, el inadecuado tratamiento de los residuos, constituyen factores de riesgo de enfermedades parasitarias y son de impacto relevante en los sectores sociales más carenciados o vulnerables (Bolzan y col., 2005; Pezzani y col., 2012)

En Argentina existe registro de la situación parasitaria, realizado a través de estudios descriptivos que han aportado datos sobre prevalencia en diferentes regiones. Estos trabajos, juntamente con estudios sobre presencia de contaminantes parasitarios en sistemas geohídricos y factores socioculturales asociados a la presencia de una determinada parasitosis en una región particular, dan una aproximación de la 
realidad del fenómeno parasitario local (Gamboa y col., 2011). Esta información es necesaria como punto de partida para avanzar y contribuir al cambio de una situación sanitaria local, así como revertir un contexto desfavorable. Para ello, se deben ejecutar acciones de control que eviten la transmisión y la diseminación de enfermedades de etiología parasitaria, mejorando así la calidad de vida de las personas y su propio desarrollo socioeconómico.

Nuestro equipo de trabajo comienza a trabajar en parasitosis intestinales en el año 2002, en el marco de proyectos de investigación subsidiados por la Universidad Nacional de La Plata (UNLP). Se implementó el trabajo de campo de estas infecciones en comunidades vulnerables de Municipios del noroeste de la provincia de Buenos Aires, Argentina. Nos enfocamos especialmente en determinar fuentes de infección, prevalencias, asociaciones con variables ambientales y socioculturales, así como determinación de factores de riesgo (Pezzani y col., 2009, 2012; Molina y col., 2011; Orden y col., 2014; Ciarmela y col. 2016; Minvielle y col. 2016).

Esta información, con significancia epidemiológica, nos llevó a replantear el verdadero propósito de los proyectos de Investigación y anexar la Extensión subsidiada por la UNLP. La intención fue bien definida: no quedarse con el dato de prevalencia; ir por más, avanzar y analizar estrategias que pudieran modificar la realidad sanitaria teniendo en cuenta el contexto social, cultural y geográfico. Que esta problemática, desatendida por las autoridades sanitarias, pudiera a través de nuestro recorrido llevar el tema a la comunidad; que se hablara de las parasitosis en los niños y se tomara conciencia de la importancia como enfermedad crónica que lenta y gradualmente va deteriorando al organismo y afectando el desarrollo pondoestatural y capacidades cognitivas. Luego de instalado el tema, difundir medidas de control que la comunidad pudiera realizar y mejorar especialmente las condiciones sanitarias.

Posteriormente, con la incorporación de una antropóloga al equipo, comenzamos la evaluación de peso, talla e índice de masa corporal. Si bien esta actividad registró deficiencias en el desarrollo pondoestatural de algunos escolares, fuimos detectando un alarmante progreso de sobrepeso y obesidad; estados que reflejan una mala calidad nutricional en estas comunidades.

En los comienzos, los docentes-investigadores-extensionistas trabajábamos con profesionales sanitarios de cada región para recabar datos epidemiológicos y tomas de muestras de los niños que consultaban en las Unidades Sanitarias, y que trabajaban en conjunto con el equipo universitario. Los docentes realizaban el diagnóstico parasitario y la Unidad Sanitaria indicaba el tratamiento a cada escolar. Posteriormente, el equipo universitario capacitaba a referentes comunitarios para realizar las tareas de promoción y prevención de las parasitosis detectadas y de las deficiencias alimentarias.

En 2009, la UNLP otorga carácter de PROGRAMA de Control de las Parasitosis Intestinales y Nutrición (PROCOPIN por sus siglas), adquiriendo el mismo una relevancia institucional. A partir de ese año, se realiza la convocatoria a estudiantes de la Facultad de Ciencias Médicas de la UNLP, para participar en las diferentes etapas que se diseñaron en este programa. En 2012, la Facultad de Ciencias Médicas de la UNLP lo acredita como Programa Estable de la mencionada Facultad, jerarquizando sus actividades.

La continuidad se ha asegurado hasta la fecha a través de acreditaciones y subsidios obtenidos en Convocatorias de Extensión y de Investigación, y la articulación con la Subsecretaría de Políticas Sociales de la UNLP quien coordina el trabajo territorial 
a través de los Centros Comunitarios de Extensión Universitaria. Este Programa se ha implementado en diferentes barrios de los Municipios de La Plata, Berisso y Magdalena, situados al noreste de la provincia de Buenos Aires. Se contacta con la comunidad a través de los establecimientos escolares de ámbito urbano, suburbano y rural, seleccionados por la demanda de los profesionales educativos, sanitarios y referentes comunales de cada región. Actualmente, el equipo que desarrolla las diferentes actividades está conformado por médicos, biólogos, bioquímicos, antropólogos y veterinarios docentes-investigadoresextensionistas pertenecientes a las Facultades de Ciencias Médicas (responsables), Ciencias Naturales y Museo y Ciencias Veterinarias de la UNLP, otorgándole a la temática sobre las parasitosis intestinales y alteraciones de la nutrición un enfoque amplio dado por la integración de disciplinas. Las diferentes miradas sobre esta problemática ofrecen la posibilidad concreta y efectiva de modificar una situación común a personas en estado de vulnerabilidad social y mejorar su calidad de vida. También actualmente, la participación de los estudiantes universitarios como voluntarios de PROCOPIN involucra no sólo a estudiantes de la Facultad de Cs. Médicas, sino también de las otras Facultades participantes.

PROCOPIN se desarrolla en etapas: 1- Evaluación del estado socio-sanitario, nutricional y parasitario de escolares (3-12 años), 2-Intervención terapéutica en niños; tratamiento de mascotas e intervención ambiental factible 3- Talleres educativos y 4Control post-intervención.

\section{Objetivos y Metas de PROCOPIN:}

Objetivo General: mejorar la salud y calidad de vida de las personas de comunidades vulnerables de nuestra región.

Objetivos Específicos:

1- Trabajar con comunidades de elevada prevalencia parasitaria y alteraciones de la nutrición, analizando los factores de riesgo propios de cada localidad.

2-Implementar estrategias de prevención y control de las patologías mencionadas.

3-Evaluar el impacto de las actividades realizadas.

\section{Metas propuestas:}

Disminución de la prevalencia de parasitosis intestinales en la comunidad.

Disminución de los trastornos de nutrición y de la anemia en los niños.

Adopción de correctos hábitos de higiene por parte de sus habitantes.

Concientizar a la comunidad sobre el potencial zoonótico de los parásitos de mascotas.

Disminución de la contaminación parasitaria del ambiente donde reside la comunidad.

Integración de los estudiantes universitarios con la comunidad.

Difusión de los resultados. 


\section{Actividades}

Actividad 1: Se realizan reuniones convocadas por los Directivos de la Escuelas y del Jardín de Infantes del barrio seleccionado. A estas reuniones asisten los padres/ tutores de los nińos. El equipo de trabajo explica los objetivos del proyecto. Se realiza una encuesta voluntaria individual para evaluar características socio-culturales y clínicas de cada uno de los escolares (con participación de estudiantes universitarios). Se les entrega los recipientes de toma de muestra para el estudio parasitológico con las indicaciones para su realización a quienes deseen participar del proyecto. Por experiencias anteriores, los padres y/o tutores de los niños consideran que sus hijos no están parasitados porque no evidencian síntomas agudos de enfermedad. Incluso ellos mismos se consideran libres de parásitos. Al informarles que el análisis es totalmente gratis y voluntario y que de esta manera se aseguran que no tienen parásitos, la convocatoria es importante. De esta manera se logra la concientización de los padres sobre la presencia de niños parasitados en la comunidad.

Actividad 2. Se recolectan las muestras, se procesan y se elabora un informe que se entrega a cada padre/tutor. Se toma una muestra de sangre (voluntaria, con consentimiento informado y acompańamiento del padre/tutor) para evaluación de anemia y nutrientes esenciales. También se realiza el registro de peso y talla e índice de masa corporal para evaluar desnutrición/sobrepeso/obesidad, según recomendaciones de la Organización Mundial de la Salud. Se recolectan muestras del agua de los establecimientos y de la tierra donde se asienta la comunidad. Se determina su grado de contaminación biológica. Se detectan mascotas parasitadas, mediante entrega de recipientes de toma de muestra y posterior observación microscópica.

Actividad 3: Se completa una base de datos con todos los registros efectuados. Se analizan las asociaciones entre las parasitosis encontradas, la contaminación ambiental y de animales, anemia, alteraciones de la nutrición y las características socioculturales y clínicas de cada uno de los encuestados. Se seleccionan las estrategias de intervención, en base a esta información.

Actividad 4: Se ofrece tratamiento antiparasitario gratuito a las personas parasitadas quienes deben concurrir al Centro de Salud cercano al establecimiento escolar, para su tratamiento individual y selectivo. También es gratuito el tratamiento para los casos de anemia.

Actividad 5: se convoca a los estudiantes universitarios para participar activamente en los encuentros de promoción de la salud en la comunidad. Los docentes capacitan a los estudiantes sobre la problemática parasitaria (tanto endo como ectoparasitosis) y la manera de evitar la transmisión con cambios en hábitos factibles en la comunidad. Con el asesoramiento de una Licenciada en Nutrición se capacita a los estudiantes sobre conductas que pueden modificarse en niños, con trastornos de alimentación. Se evalúan los hábitos riesgosos (no saludables) y saludables registrados en la comunidad, se identifican las fuentes y vías de transmisión de las parasitosis, los reservorios parasitarios y las barreras que pudieran contrarrestar la diseminación parasitaria. Se establecen cuáles serán las modificaciones necesarias para generar un cambio de situación, que constituirán los mensajes fundamentales en cada encuentro educativo con la comunidad. Se realizan los talleres interactivos. 
Actividad 6: Se realiza un control post-intervención de las parasitosis en 30\% de las personas que participaron en la primera etapa. Se vuelve a realizar la encuesta sociocultural y clínica, para registrar los cambios producidos en las conductas del $30 \%$ de las personas que participaron en la primera etapa

Actividad 7: se realiza un informe final que se eleva a las autoridades sanitarias de la región.

\section{Consideraciones éticas para proyectos que incluyen sujetos humanos}

Información oral que se comunica en las reuniones:

Nos presentamos, les preguntamos si saben lo que son los parásitos y luego se informa lo siguiente: La UNLP, el Centro de Salud del barrio y esta Escuela/Jardín de Infantes estamos desarrollando un proyecto de control de las parasitosis intestinales y nutrición. Contamos con la colaboración de estudiantes de Medicina y de Ciencias Naturales que se interesaron en trabajar con esta comunidad. Mediante este proyecto intentaremos disminuir las parasitosis intestinales, que provocan alteraciones en el crecimiento y en la capacidad de aprender de los niños de esta escuela. Para ello, les pedimos su colaboración. Haremos una encuesta y tomarán muestras de materia fecal de sus niños para que nosotros las podamos estudiar (aquí les mostramos los recipientes). Todos los datos que registremos serán confidenciales, eso quiere decir que solamente ustedes sabrán qué parásitos tiene su hijo/a. El informe se entrega en sobre cerrado con el nombre del padre/tutor. Para evaluar el crecimiento, los mediremos y pesaremos (aquí les mostramos los elementos que usaremos para ello). A veces, algunos parásitos provocan anemia y cambios en minerales de la sangre, que les produce cansancio. Para medirlos, necesitamos sacar sangre del brazo con aguja y jeringa estéril. Para los que autoricen este estudio, la extracción la realizará una bioquímica: Marina (la presentamos) que tiene mucha experiencia en esta práctica. Pueden preguntar todo lo que quieran saber. Todo es gratuito y voluntario y los resultados se entregan en sobre cerrado. Todos los nińos que resulten parasitados y/o con anemia y/o con alteraciones en algunos minerales serán tratados gratuitamente en la Unidad Sanitaria. En la segunda mitad del ańo, volveremos para dar charlas sobre cómo prevenir la infección de los parásitos detectados y mejorar los hábitos alimenticios con medidas que son posibles de realizar hasta por los propios nińos. Todos los análisis que nosotros hacemos deben contar con la autorización firmada por el padre o tutor del niño al final de la encuesta. Para la extracción de sangre, el padre/ madre o tutor debe acompañar al niño. Para permitir que los midamos y pesemos sólo se requiere la autorización firmada. ¿Quieren preguntarnos algo?

\section{Resultados:}

\section{Extensión:}

Desde su concepción, el Programa impacta fuertemente en el ámbito de la comunidad estudiantil que participa de manera entusiasta y comprometida; y en las esferas de la UNLP, con gran reconocimiento a través del apoyo de la Secretaría de Extensión y de la Subsecretaría de Políticas Sociales, para participar en diferentes eventos 
afines a la actividad territorial. Del mismo modo, PROCOPIN contó con la aprobación y conformidad de la Secretaría de Extensión de la Facultad de Ciencias Médicas (UNLP) y el apoyo de todas las comunidades donde fue implementado; abriendo la posibilidad de que una realidad sanitaria desfavorable comience a girar hacia una situación de mayor bienestar, y mejor calidad de vida.

Desde PROCOPIN se han desarrollado estrategias de intervención con resultados alentadores. Estas estrategias tienen en cuenta la realidad económica y cultural de la población parasitada. No nos quedamos "esperando al enfermo", sino que acudimos a la comunidad para revertir la situación. Este programa resulta de gran interés comunitario porque permite controlar la transmisión de las parasitosis intestinales en comunidades vulnerables, teniendo en cuenta que la Organización Mundial de la Salud advierte sobre los deficientes resultados que surgen de instaurar solamente el tratamiento farmacológico, si las personas no cambian sus inadecuados hábitos higiénico-sanitarios. Los niños afectados reciben tratamiento específico y gratuito a cargo de las Unidades Sanitarias de cada barrio. Posteriormente se realiza la intervención educativa desarrollada por los estudiantes de Medicina y Antropología en un trabajo colectivo rico, cargado de mensajes que posibilitan actividades escolares en espacios áulicos y recreativos. El impacto de la intervención ha sido evaluado a lo largo de estos años, con porcentajes significativos de disminución de las parasitosis y un efectivo mejoramiento de los hábitos de higiene en la comunidad.

Las actividades de extensión han sido motivo de diversas publicaciones y presentaciones en Congresos y Jornadas. Mencionamos las realizadas en los últimos ańos: -Dos ańos de PROCOPIN". Minvielle M, Pezzani B, Ciarmela L, Molina N, Orden A, Rosa D. XI Congreso Iberoamericano de Extensión Universitaria. Santa Fe, 22-25 de noviembre de 2011.

-Promoción de la Salud en los Centros Comunitarios de Extensión Universitaria, Universidad Nacional de La Plata; Argentina. Pezzani BC, Ciarmela ML, Isla Larrain M, Blas Y, Orden AB, Minvielle MC.http://formularios.extension.edu.uy/ ExtensoExpositor2013/trabajos.html

Congreso de Extensión Asociación Universidades Grupo Montevideo. Extenso 2013, Montevideo.

-PROCOPIN: la experiencia de integrar enseńanza, investigación y extensión en un proyecto de la UNLP. Pezzani BC, Ciarmela ML, Rosa D, Isla Larrain M, Apezteguía C, Martinez C, Orden B, Minvielle MC. III Jornadas de Extensión del MERCOSUR. Tandil. Abril 2014

-Acciones interdisciplinarias de post-inundación frente a las parasitosis emergentes en el barrio "El Mercadito" de La Plata a través del CCEU No 6 (UNLP). Ciarmela ML, Pezzani BC, Gamboa MI, Osen B, Butti M, Kozubsky L, Costas ME, Oliva D, Minvielle MC. VI Congreso de Extensión Universitaria. Rosario. Septiembre 2014.

-Parasitosis intestinales, toxocariasis y esosinofilia en escolares de barrios periféricos de La Plata. Orden A, Ciarmela L, Zubiri K, Pezzani B, Piacenza G, Minvielle M. VI Jornadas de extensión universitaria de investigaciones relacionadas con la salud y ciencias afines y X Jornadas de investigaciones del equipo de salud. 23 -25 de Septiembre de 2015. Santa Rosa, La Pampa. 
-Cortometraje educativo/sanitario: "Las aventuras del capitán Toxocara" Butti,M J; Minvielle,M C; Gamboa,M I. V Jornada de Extensión del MERCOSUR. 19-20 de Mayo 2016, Tandil, Argentina.

\section{Docencia}

Valorizando la vivencia formativa y significativa de los estudiantes en los proyectos de extensión que desarrollamos desde la Cátedra de Microbiología y Parasitología de la Facultad de Ciencias Médicas, hemos contado con la participación voluntaria de los estudiantes universitarios en un promedio de 40 alumnos por año. En los mencionados proyectos, el rol de los estudiantes a través de los años pasó de ser un simple observador de las tareas de intervención a tomar un papel protagónico en la implementación de las mismas; asumiendo un compromiso con la comunidad en la búsqueda de solución a problemas concretos inherentes a nuestra asignatura.

Con esta propuesta de intervención comunitaria se plantea profundizar la formación académica de los estudiantes integrando formalmente contenidos de aprendizaje, experiencias de trabajo y servicio en la comunidad con intencionalidad pedagógica de aprendizaje activo (aprender haciendo) impactando en una mejor formación integral con la producción de nuevos conocimientos, aportados por las experiencias en terreno.

Hacer investigación-extensión propone al estudiante acercarse a la comunidad para realizar promoción primaria y secundaria de la Salud, tal como recomienda la Organización Mundial de la Salud; y no esperar en un consultorio a que "llegue" el enfermo, como es la impronta actual de la carrera. Los estudiantes de Medicina se ven enriquecidos en su formación académica y humanística, además de introducirse en una de las problemáticas sanitarias de nuestra región.

Los estudiantes han difundido sus experiencias en las siguientes comunicaciones:

Experiencias de estudiantes voluntarios en PROCOPIN (Programa de Control de las Parasitosis Intestinales y Nutrición). Muñoz E, Molina Blanco C, Malmoria A, Mena J, Ciarmela M, Molina N, Pezzani B, Minvielle M. Jornadas de Medicina 2009, Fac. Cs. Médicas, UNLP, 28 y 29 de octubre.

PROCOPIN: programa de control de parasitosis intestinales y nutrición. Nuestra experiencia como estudiantes voluntarios. Vidal V, Pappalardo V, Prenollio Riddick ME; Rodríguez M, Ciarmela ML, Molina NB, Pezzani BC, Minvielle MC: Jornadas Medicina. La Plata, octubre 2011.

Comunidad y universidad: control de parasitosis intestinales y estado nutricional. Beretta N, Duboscq V; Falcone A; Oronó M; Perri D; Saiz Rodriguez D; Sanchez R; Simonetti R; Stafeta N; Torres S; Vertua E; Ciarmela L; Pezzani B; Minvielle M. V Congreso de Extensión Universitaria, Córdoba, 10-12 Septiembre 2012

\section{Investigación.}

El análisis de los resultados obtenidos ha sido plasmado en numerosos trabajos y presentaciones en reuniones científicas. Mencionamos los publicados en los últimos años: 
- Toxocariosis: perfil hematológico y concentración sérica de calcio, magnesio, zinc y cobre en escolares de Berisso. Molina N, Ciarmela ML, Pezzani B, Apezteguía M, Rosa D, Minvielle M. Io Congreso Internacional de Zoonosis y Enfermedades Emergentes. VII Congreso Argentino de Zoonosis. Buenos Aires, junio de 2011.

- Factores de riesgo en la transmisión de giardiosis en el ámbito rural. Pezzani B, Ciarmela ML, Apezteguia M, Molina N, Minvielle M. Io Congreso Internacional de Zoonosis y Enfermedades Emergentes. VII Congreso Argentino de Zoonosis. Buenos Aires, junio de 2011.

- Situación nutricional y parasitosis en nińos escolares de Berisso. Molina N, Rosa D, Pezzani B, Ciarmela ML, Orden B, Apezteguía M, Minvielle M. Jornadas Medicina. La Plata, octubre 2011.

- Intestinal parasitoses in suburban and rural schoolchildren in Argentina. Pezzani B, Ciarmela ML, Apezteguía M, Molina N, Rosa D, Orden A, Minvielle M. Rev Pat Trop (ISSN 0301-0406) 41: 63-73(2012).

- Transmisión de las Parasitosis intestinales y Conductas sociales. Pezzani BC, Ciarmela ML, Apezteguía MC, Orden AB, Rosa D, Minvielle MC. VII Congreso de la Sociedad argentina de Bacteriología, Micología y Parasitología Clínicas. SADEBAC. CABA, 26 al 29 de Junio de 2012. Revista de la Asociación Argentina de Microbiología Supl. 1, Vol 44, pág. 87, 2012

- Toxocariasis, eosinofilia y parasitosis intestinales en escolares de la provincia de Buenos Aires, Argentina" Ciarmela M, Videla L, Pezzani B, Apezteguía M, Molina N, Minvielle M. III Jornada Platense de Salud Pública, Enfermedades Emergentes y Zoonóticas. I Jornada sobre Cambio Global y Desarrollo Sustentable. La Plata, Junio 2012. Revista de Enfermedades Infecciosas Emergentes, vol 7, 2012-2013, pág. 15.

- Estrategias de control de las parasitosis intestinales en una comunidad rural de la provincia de Buenos Aires. Pezzani B, Ciarmela ML, Apezteguía M, Orden B, Rosa D, Isla Larrain M, Minvielle M. III Jornada Platense de Salud Pública, Enfermedades Emergentes y Zoonóticas. I Jornada sobre Cambio Global y Desarrollo Sustentable. La Plata, Junio 2012. Revista de Enfermedades Infecciosas Emergentes, vol 7. 2012-2013, pág. 22.

- Perfil nutricional y parasitosis en escolares de zona rural de Magdalena, provincia de Buenos Aires" Molina N, Ciarmela L, Isla Larrain M, Orden B, Apezteguía M, Pezzani B, Minvielle M. IIo Encuentro Nacional de Epidemiología Pediátrica. Sociedad Argentina de Pediatría. 19/10/2012. CABA.

- Salud nutricional y enteroparasitosis: relevamiento comunitario en dos distritos bonaerenses". Orden A, Apezteguia M, Ciarmela M, Molina N, Pezzani B, Rosa D, Minvielle M. Congreso latinoamericano de Salud Pública 2012. 28-30 Noviembre 2012. Córdoba

- Toxocariasis in schoolchildren in urban and rural areas of Argentina”. Molina Nora. Colaboradora: Minvielle Marta. International Congress on Bacteriology and Infectious Diseases. Baltimore. Estados Unidos de Norte América. 2013.

- Eosinofilia y factores de riesgo personales asociados a toxocariasis en nińos escolares de la provincia de Buenos Aires". Pezzani B, Ciarmela ML, Isla Larrain M, Apezteguía M, Molina N, Minvielle M. XIII Congreso Argentino de Microbiología- II Congreso Microbiología Agrícola y Ambiental CABA, 2013. 
- Toxocariasis encubierta autolimitada en escolares de La Plata, Argentina”. Ciarmela ML, Pezzani B, Isla Larrain M, Blas Y, Minvielle M. I Congreso Internacional de la Facultad de Ciencias Médicas de la Universidad Nacional de La Plata. 28-30 Noviembre, 2013

- Nutritional Status in Parasitized and Nonparasitized Children from Two Districts of Buenos Aires, Argentina. Orden A, Apezteguía M, Ciarmela ML, Molina N, Pezzani B, Rosa D, Minvielle M. Published on line Noviembre 2013 in Wiley Online Library. American Journal of Human Biology 26: 73-79 (2014).

- Protozoosis en escolares de una comunidad semirural de la provincia de Buenos Aires. Ciarmela ML, Ceccarelli S, Zubiri K, Pezzani B, Minvielle M. XX Jornada sobre Enfermedades Infecciosas Emergentes y Reemergentes. XVIII Cambio Global y Desarrollo Sostenible. 12 de Noviembre de 2015. La Plata.

- Abordaje multidisciplinario de patologías transmisibles y nutricionales en escolares de La Plata y Berisso articulando investigación, extensión y docencia. Minvielle M, Pezzani B, Ciarmela ML, Orden A, Lareschi M, Isla Larrain M, Martinez C, Rosa D, Marińelarena A, Zubiri K, Ceccarelli S, Bernal V, Jorge S, Piacenza G. XX Jornada sobre Enfermedades Infecciosas Emergentes y Reemergentes. XVIII Cambio Global y Desarrollo Sostenible. 12 de Noviembre de 2015. La Plata.

- Parásitos zoonóticos en mascotas caninas y felinas de escolares suburbanos Piacenza G, Jorge S, Zubiri K, Ceccarelli S, Ciarmela L, Pezzani B, Minvielle M. II Congreso Internacional de Medicina. 12-14 de Noviembre de 2015. La Plata. Argentina.

- Anemia in children aged 4 to 8 from a semirural community in central east area of Argentina. Ciarmela ML, Pezzani BC, Isla Larrain M, Martínez CP, Apezteguía MC, Minvielle MC. World Health \& Population. 16(3) March 2016: 22-30.doi:10.12927/ whp.2016.24517

- Toxocariasis, intestinal parasitoses and eosinophilia in schoolchildren from Argentina. Ciarmela ML, Pezzani B, Minvielle M. Current Health Sciences Journal 42 (1): 5-11: 2106. ISSN 2067-0656. ISBM: 2069-4032.

- Nutritional hematic parameters and presence of parasitic infection in children from urban environment in La Plata, Argentina. Minvielle M, Ceccarelli S, Zubiri K, Ciarmela L, Rosa D, Pezzani B, Bonzo E. Ponte Journal (International Scientific Researches Journal) 72 (2): 15-160: 2016.ISSN 0032-423-X. E-ISSN: 0032-6356.

\section{Desafío actual}

Es indudable que PROCOPIN ha crecido y expandido en el territorio tomando una dimensión diferente a la original, y transformándose así en una vertiente que brinda conocimiento y experiencia a los estudiantes, posibilidad de mejorar la salud y calidad de vida a las personas de los barrios donde arribamos con nuestra propuesta; y estímulo para los docentes que creemos y apostamos a la integración de la docencia-investigaciónextensión. Propiciado por la Facultad de Ciencias Médicas y la UNLP el equipo de trabajo es invitado todos los ańos para difundir sus actividades en el stand correspondiente en la EXPO-UNLP, donde se promocionan las carreras y proyectos de todas las facultades. También ha sido motivo de numerosas entrevistas periodísticas publicadas en periódicos 
de la región y en programas radiales. En 2011, PROCOPIN fue seleccionado por la UNLP como proyecto de Voluntariado Universitario (Secretaría de Política Universitaria) para participar del programa televisivo "Experiencia Directa" del Canal Encuentro de Televisión por cable.

En este amplio recorrido de crecimiento y desarrollo fueron inevitables objeciones, críticas y resistencias por parte de docentes, un minúsculo grupo integrado por investigadores que entienden su función científica como la única y trascendental aspiración. En este contexto, nuestro desafío actual es seguir avanzando con la convicción que el camino encarado es el correcto, enfocados en nuestros objetivos, desestimando la mirada contraria y bregando para que más docentes y estudiantes entusiastas distingan en esta integración una superadora propuesta académica.

\section{Discusión}

Pobreza y mala salud son fenómenos interrelacionados. Los países pobres tienden a presentar inferiores indicadores sanitarios que los más desarrollados, y dentro de cada país las personas pobres tienen más problemas de salud que las más ricas. Esta asociación refleja una relación de causalidad que funciona en los dos sentidos: la pobreza genera mala salud, y la mala salud hace que los pobres sigan siendo pobres (Wagstaff, 2002).

Se sabe muy poco sobre la importancia relativa de las desigualdades con respecto a los factores determinantes de la salud, así como la utilización de los servicios de salud. Lo que sabemos indica que las desigualdades sanitarias, y muy probablemente también la utilización de los servicios, reflejan en gran medida las desigualdades con respecto a variables tanto individuales como familiares, tales como la educación, los ingresos, la localización y las características de los hogares. La importancia de la alimentación y nutrición constituyen uno de los determinantes fundamentales de la salud (Cantú Martínez, 2005)

En nuestra región aún existe un elevado número de personas que se encuentran en situación de pobreza y vulnerabilidad. A esto se suma el acceso deficiente a los servicios básicos de salud, educación, vivienda, suministro de agua y saneamiento. Esto trae aparejado el surgimiento de enfermedades infecciosas, tal es el caso de las parasitosis emergentes y reemergentes. La falta de percepción de riesgo comunitario dificulta la prevención de estas patologías.

Las autoridades sanitarias de nuestra región han manifestado su preocupación por las parasitosis presentes en personas que viven en condiciones precarias, principalmente en niños de edad escolar de barrios periféricos. Estas patologías son de curso crónico, y deterioran lentamente al individuo de tal manera que no percibe la afectación de su organismo, hasta que alcanza niveles muchas veces irreversibles. Debido a la condición de cronicidad y lento deterioro, estos niños no son llevados a la consulta médica en consultorios de Unidades Sanitarias cercanas a su asentamiento. Estas características definen la importancia de desarrollar programas que ingresen a la comunidad, detecten las patologías e implementen su tratamiento; de manera tal que evitan la progresión de las mismas.

Detectar factores de riesgo y trabajar en la prevención de aquellos modificables, mediante el cambio de conductas higiénico-sanitarias, es un desafío para la salud pública 
más aún en sectores vulnerables, con difícil acceso al sistema de salud. En este marco, se desarrolla este Programa, que cumple un rol relevante al no contar en Salud Pública con algún organismo que implemente este control.

El objetivo educativo de la carrera de Medicina de la Facultad de Ciencias Médicas es la formación de un médico general, definido como "el profesional que está capacitado en conocimientos, habilidades y actitudes necesarias, saber desarrollar estrategias de aprendizaje autónomo que le permitan capacitarse en una ulterior especialización de postgrado, que el medio y su inquietud personal le reclamen".

La propuesta de PROCOPIN ha resultado de interés académico porque la formación extramuros no hospitalaria es escasa en la currícula de la carrera de Medicina, constituyendo un desafío para el estudiante de Medicina. Desde este enfoque abarcador y desafiante que estimula a tomar herramientas conceptuales de distintas disciplinas, iniciamos un programa de trabajo compartido entre docentes de las facultades de Cs. Médicas, Cs. Veterinarias y Cs. Naturales.

En este escenario, entendemos decisivo desarrollar metodologías de enseñanza acordes a este modelo curricular basado en la adquisición de competencias. La participación de los estudiantes en nuestras actividades en la comunidad, brinda la oportunidad de involucrarse en la resolución de problemas reales, trabajando en escenarios complejos, en los que se debe poner a prueba el conocimiento académico, la ínter disciplina y la interacción con otros actores sociales. El provenir de carreras con distintas áreas de incumbencia, evidentemente permitirá que el trabajo sea atravesado por docentes y alumnos desde la mirada UN MUNDO = UNA SALUD (Garza Ramos, 2012).

Así lo entendemos y así es cómo resuena en los estudiantes que año a año han participado activos, creativos en PROCOPIN. Ellos encuentran sentido a su aprendizaje, se motivan, los estimula y alienta, se sienten involucrados. La participación de los estudiantes en los proyectos de extensión constituye una opción más en los procesos de enseñanza y aprendizaje, es formativo. Compartimos la idea que estas experiencias de prácticas impactan consistentemente en la comprensión y aplicación de los conocimientos, en el desarrollo de interés por indagar, del pensamiento reflexivo y crítico.

De nuestra experiencia se desprende que las vivencias experimentadas por los estudiantes en PROCOPIN tienen un significativo valor formativo. La Universidad se reconoce como parte de la Comunidad, y nosotros la entendemos como un espacio donde se aprende, se investiga, se construyen alianzas institucionales, y donde las iniciativas solidarias llevadas a cabo junto con la comunidad son para estudiantes y docentes también una forma de aprender y de investigar. La práctica educativa asume un perfil participativo y colaborativo, en la cual la investigación se plantea como un análisis crítico orientado hacia la transformación de las propias prácticas (Dubrovsky y col., 2003).

Respecto a la investigación, los resultados obtenidos han sido objeto de presentaciones en reuniones del área científica y publicaciones en revistas nacionales y extranjeras. El conocimiento acerca de la distribución y prevalencia de las infecciones parasitarias y alteraciones nutricionales en niños, es insuficiente en nuestro país; pues los servicios de salud y asistencia médica le conceden precarios recursos para su estudio y control epidemiológico.

La mayoría de las enfermedades parasitarias y alteraciones nutricionales no son de denuncia obligatoria, y en general ocurren en poblaciones marginales de las ciudades o en zonas rurales carentes de servicio de asistencia médica y de salud, lo que dificulta su 
diagnóstico etiológico. Por lo tanto, las estadísticas de morbilidad y de mortalidad no reflejan la realidad. El aporte de PROCOPIN en esta problemática se considera relevante, ya que trabaja con la comunidad escolarizada que es cercana al 97\% en Argentina (http:// publicaciones.siteal.org/perfiles-de-pais/4/argentina)

Los datos epidemiológicos aportados por PROCOPIN a las autoridades sanitarias de la región, pueden influir en los niveles de decisión política y en las instituciones nacionales para logar una disminución de la pesada carga que las parasitosis y las alteraciones nutricionales aún significan, para la salud y la vida de extensos sectores de la población.

Respecto a las diferencias y desacuerdos con docentes-investigadores, nos hemos propuesto resistir a la idea de la supremacía de la Investigación sobre la Extensión; promoviendo eventos dentro del ámbito de la Facultad de Cs. Médicas. En los mismos se convoca a todos los docentes, investigadores y extensionistas para exponer propuestas, resultados, nuevos desafíos y experiencias en la integración de la extensión-docencia e investigación. El objetivo es lograr que los docentes-investigadores enmarquen a la extensión en una nueva dimensión, y se sumen a su reconocimiento y resignificación.

\section{Conclusiones}

Este modelo de programa refleja la unión entre la institución universitaria, los profesionales de la salud pública regional y los directivos de establecimientos escolares, para lograr el control de las infecciones parasitarias intestinales y mejorar las condiciones nutricionales de niños en condiciones de vulnerabilidad social.

Esta propuesta en el contexto interno será controvertida, pero entendemos interesante el debate que pueda darse más allá del resultado para compatibilizar posiciones y suavizar diferencias. Será una oportunidad de entendimiento, de abrir posturas, debatir ideas, con el denominador común puesto en la intención de mejorar la enseńanza y avanzar en un proyecto, que lleve respuestas a demandas concretas de las poblaciones desprotegidas.

Las "Prácticas Educativas Solidarias en Educación Superior" son una de las tantas expresiones de un nuevo paradigma, que ya está en práctica en muchas partes del mundo (Tapia, 2008). En este modelo, la Universidad se reconoce como "parte de" la comunidad.

De esta manera, la comunidad es vista como un espacio donde se aprende, se investiga, se construyen alianzas institucionales, y donde las iniciativas solidarias llevadas a cabo junto con la comunidad son para estudiantes y docentes también una forma de aprender y de investigar.

\section{Referencias bibliográficas}

BOLZAN, A.; MERCER, R.; RUIZ V.; BRAWERMAN, J.; MARX, J.; ADROGUE, G.; CARIOLI, N.; CORDERO, C. Evaluación nutricional antropométrica de la niñez pobre del norte argentino: Proyecto encuna. Archivos Argentinos de Pediatría 103(6): 545-555: 2005.

CANTÚ MARTINEZ, P. C. Alimentación, nutrición y estrategias en salud pública. VII Congreso Regional de Químicos Farmacéuticos Biólogos San Nicolás de los Garza, N.L. México17 al 20 de Mayo, 2005. 
CIARMELA, M. L.; PEZZANI, B. C.; ISLA LARRAIN, M.; MARTÍNEZ, C. P.; APEZTEGUÍA, M. C.; MINVIELLE, M. C. Anemia in Children Aged 4 to 8 from a Semirural Community in Central East Area of Argentina World Health \& Population. Vol. $16 \mathrm{~N}^{\circ} 3,2016$.

CULHA, G.; SANGUN, M. Serum levels of zinc, copper, iron, cobalt, magnesium, and selenium elements in children diagnosed with Giardia intestinalis and Enterobius vermicularis in Hatay, Turkey. Biology Trace Elements Research 118: 21-26: 2007.

DUBROVSKY, S.; IGLESIAS, A.; SAUCEDO, E. La reflexión sobre las prácticas docentes a partir de un proyecto de investigación-acción. Educación, Lenguaje y Sociedad 1: 111-119:2003.

GAMBOA, M.; NAVONE, G.; ORDEN, A.; TORRES, M.F.; CASTRO, L.E.; OYHENART, E. E. Socio-environmental conditions, intestinal parasitic infections and nutritional status in children from a suburban neighborhood of La Plata, Argentina Acta Tropica 118: 184-9:2011.

GARZA RAMOS, J; ARVIZU TOVAR, L. Hacia una salud: Propuesta en el marco de la Administración Pública Federal en México. Editorial Yire. $1^{\circ}$ Ed México. 2012.

HOTEZ, P.J.; BOTTAZZI, M.E.; FRANCO-PAREDES, C.; AULT, S.K.; PERIAGO, M.R. The Neglected Tropical Diseases of Latin America and the Caribbean: A Review of Disease Burden and Distribution and a Roadmap for Control and Elimination. PLoS Neglected Tropical Disease 2(9): 300:2008.

MINVIELLE, M.; CECCARELLI, S.; ZUBIRI, K.; CIARMELA, M.L.; ROSA, D.; PEZZANI, B.; BONZO, E. Nutritional hematic parameters and presence of parasitic infection in children from an urban environment in La Plata, Argentina. International Scientific Researches Journal, Vol. 72 (2): 150-160:2016.

MOLINA, N.; PEZZANI, B.; CIARMELA, M.L.; ORDEN, A.; ROSA, D.; APEZTEGUÍA, M.; Basualdo, J.; MINVIELLE, M. Intestinal parasites and genotypes of Giardia intestinalis in school children from Berisso, Argentina. Journal of Infectious in Development Countries; 5(7):527-534:2011.

ORDEN, A.B.; APEZTEGUÍA, M.C.; CIARMELA, M.L.; MOLINA, N.B.; PEZZANI, B. C.; ROSA, D.; MINVIELLE, M. C. Nutritional Status in Parasitized and Nonparasitized Children from Two Districts of Buenos Aires, Argentina. American Journal of Human Biology 26:73-79: 2014.

PEZZANI, B. C.; MINVIELLE, M. C.; CIARMELA, M.L.; APEZTEGUÍA, M.C.; Basualdo, J. A. Participación comunitaria en el control de las parasitosis intestinales en una localidad rural de Argentina. Revista Panamericana de Salud Pública 26(6):471-477: 2009.

PEZZANI, B.; CIARMELA, M.L.; APEZTEGUÍA, M.C.; MOLINA, N.; ORDEN, A.; ROSA, D.; MINVIELLE, M. Intestinal Parasitoses in Suburban and Rural Schoolchildren in Argentina. Revista de Patología Tropical 41: 63-73. 2012.

TAPIA, N. Aprendizaje y servicio solidario en la misión de la educación superior. En: EUDEBA (1 ra. Ed). El Aprendizaje-Servicio en la Educación Superior. Una mirada analítica desde los protagonistas. Cap. 1:11-35:2008.

WAGSTAFF, A. Pobreza y desigualdades en el sector de la salud. Revista Panamericana de Salud Pública 11:5/6:2002. 